\title{
Folate-targeted polymeric micelles loaded with superparamagnetic iron oxide as a contrast agent for magnetic resonance imaging of a human tongue cancer cell line
}

\author{
MIN-YI CUI ${ }^{1}$, ZHI DONG ${ }^{2}$, HUASONG CAI $^{2}, \mathrm{KUN} \mathrm{HUANG}^{2}, \mathrm{YI} \mathrm{LIU}^{1}$, \\ ZHUANGNIAN FANG ${ }^{2}$, XIANGMIN LI $^{2}$ and YANJI LUO ${ }^{2}$ \\ ${ }^{1}$ Department of Radiology, Hospital of Stomatology, Guanghua School of Stomatology, Sun Yat-Sen University, \\ Guangdong Provincial Key Laboratory of Stomatology, Guangzhou, Guangdong 510055; ${ }^{2}$ Department of Radiology, \\ The First Affiliated Hospital, Sun Yat-Sen University, Guangzhou, Guangdong 510080, P.R. China
}

Received May 29, 2016; Accepted April 10, 2017

DOI: $10.3892 / \mathrm{mmr} .2017 .7565$

\begin{abstract}
The aim of the present study was to investigate the feasibility of using folate-targeted superparamagnetic iron oxide nanoparticles (SPIO) as a magnetic resonance (MR) contrast agent that targets human tongue cancer cells. Folate-targeted and folate-free bilayer micelles composed of a diblock copolymer of polyethylene glycol (PEG) and poly-caprolactone (PCL) that encapsulated SPIO in their hydrophobic core (SPIO-PEG-PCL micelles) were synthesized. The cytotoxicity of each set of micelles towards Tca- 8113 cells was examined using methyl thiazolyl tetrazolium (MTT) assays. Tca- 8113 cells were incubated with folate-free SPIO-PEG-PCL micelles and folate-targeted SPIO-PEG-PCL micelles at an Fe concentration of $80 \mu \mathrm{g} / \mathrm{ml}$ for $0.5,1$ and $2 \mathrm{~h}$. MR imaging was subsequently performed and relative $T_{2}$ relaxation time was recorded. Endocytosis of each micelle type was observed using Prussian blue staining. The MTT assays demonstrated that varying concentrations of folate-targeted SPIO-PEG-PCL micelles did not result in statistically significant differences in Tca-8113 cell viability when compared with folate-free SPIO-PEG-PCL micelles $(\mathrm{F}=0.698 ; \mathrm{P}=0.676)$. In the $\mathrm{MR}$ images obtained, decreased $\mathrm{T}_{2}$-weighted signal intensity was observed for the folate-targeted SPIO-PEG-PCL and folate-free SPIO-PEG-PCL micelle treatments, particularly after the 2-h incubation period. However, the folate-targeted micelles exhibited a significantly greater decrease in signal intensity and a higher relative $\mathrm{T}_{2}$ relaxation time at each time
\end{abstract}

Correspondence to: Dr Xiangmin Li or Dr Yanji Luo, Department of Radiology, The First Affiliated Hospital, Sun Yat-Sen University, 58 The Second Zhongshan Road, Guangzhou, Guangdong 510080, P.R. China

E-mail: lixm.1111@163.com

E-mail: luoyanji163@163.com

Key words: folate, nanomicelles, magnetic resonance imaging, superparamagnetic iron oxide point $(\mathrm{P}=0.002)$. In addition, blue intracellular particles were observed in the cells that were incubated with each type of micelle and stained with Prussian blue. However, a greater number of blue particles underwent endocytosis in the folate-targeted group. Thus, folate-targeted SPIO-PEG-PCL micelles exhibited preferential targeting of Tca-8113 cells when compared with folate-free SPIO-PEG-PCL micelles, and these results support the potential for these micelles to be used for the early diagnosis of tongue cancer.

\section{Introduction}

Tongue cancer is one of the most globally common malignant tumors in the oral and maxillofacial region $(1,2)$. However, the clinical symptoms of early tongue cancer are not readily observed, thereby rendering a timely diagnosis difficult. Typically, an imaging examination is performed to ensure accurate preoperative staging and for surgical planning of the tongue mass excision. Magnetic resonance imaging (MRI) has been widely recognized as an ideal technique in the diagnosis of tongue cancer due to the markedly high resolution of soft tissue that it provides. Generally, abnormal MRI signals are flagged as lesions. In addition, neoplastic lesions in the tongue that are located towards the oral floor are readily detected on coronal images $(3,4)$. However, it is difficult to detect potential neoplastic lesions and micrometastasis within lymph nodes using MRI, particularly neoplasms that measure $<1 \mathrm{~cm}$ in diameter (5). Therefore, the sensitivity and histological specificity of traditional MR must be improved for the effective and early diagnosis of tongue cancer. Furthermore, imaging modalities that provide functional and molecular imaging would be more advantageous when compared with traditional morphological imaging (6,7). Accordingly, advances in nano-micelle science have led to the development of a non-invasive reagent that provides molecular imaging with the potential for sequential and longitudinal monitoring. In particular, super-paramagnetic iron oxide nanoparticles (termed SPIO) have been demonstrated as a highly effective contrast agent for MRI (8). Specifically, SPIO provide high magnetic signal strength and relatively low cytotoxicity $(9,10)$. However, 
crude SPIO lack specificity towards a pathological site, which potentially limits their applications. Furthermore, SPIO have a highly hydrophobic surface, which may be efficiently coated with plasma components, thereby leading to the rapid endocytosis of these particles by the reticuloendothelial system (11). Therefore, surface modification of SPIO is essential for most bio-associated applications.

To date, various nano-sized particles, including liposomes, micelles and vesicles, have been utilized as carriers for the delivery of SPIO to sites of interest $(12,13)$. Among these various modifications, micelles composed of polyethylene glycol (PEG) have been found to block copolymers with good biocompatibility. For example, a PEG outer shell for nanoparticles imparts a 'stealth' quality to hydrophobic SPIO, which helps to avoid potential exposure of the SPIO surface and adsorption of blood proteins (14). In addition, poly-caprolactone (PCL) has been widely investigated and has been applied to micelles due to its high colloidal stability compared with small molecular surfactants, and its enhanced solubilization $(15,16)$. Furthermore, PEG-PCL micelles provide a stable interface between their inner core and the aqueous environment, and this enhances the biocompatibility and biodegradability of these micelles to improve their efficacy $(17,18)$. In the present study, polymeric micelles were designed, which incorporate a diblock copolymer of PEG and PCL. The PEG-PCL block copolymers form bilayer micelles that are able to encapsulate SPIO into their hydrophobic cores $(14,15)$.

Malignant cells, due to their high rate of cell division, have an increased requirement for folate, as it is as an essential component of single-carbon metabolism and nuclear glucoside synthesis. Accordingly, the folate receptor has been found to be overexpressed by a wide range of malignant tissues, including ovarian, breast, oropharyngeal and liver cancer tissues (15-17,19-21). Furthermore, the binding efficacy of folate to the folate receptors expressed by tumor cells is high, which renders folate an ideal candidate for the targeted delivery of vehicles to tumor cells. To date, folic acid (FA) has been applied as a targeting ligand in tumor imaging diagnosis and cancer chemotherapy studies $(15,19,22)$.

To the best of our knowledge, only a small number of studies have described the targeted imaging of tongue cancer (19-23). Therefore, in the present study, folate was attached to the surface layer of SPIO-PEG-PCL copolymers to achieve functional micelles that target tongue cells. The aim was to produce SPIO-loaded micelles that would represent a $\mathrm{T}_{2}$-negative contrast agent, which could be detected by MRI to evaluate their tumor targeting efficiency noninvasively, thereby leading to the development of a method for the early detection of tongue cancer.

\section{Materials and methods}

Material preparation and characterization. Monomethoxy PEG and PCL were purchased from Sigma-Aldrich (Merck KGaA, Darmstadt, Germany). Copolymers of PEG-PCL and FA-PEG-PCL were synthesized via multistep reactions and purified according to previously reported methods $(23,24)$. The schematic illustration of folate-targeted SPIO-PEG-PCL micelle formation is presented in Fig. 1. SPIO were prepared and encapsulated inside the micelle cores as previously reported (25).
Particle sizes of the obtained nanovesicles were determined using a Brookhaven BI-200SM dynamic light scattering (DLS) instrument (Brookhaven Instruments Corp., Holtsville, NY, USA). A 532-nm vertically polarized argon ion laser was used as the light source. Measurements were made at a $90^{\circ}$ angle at $25^{\circ} \mathrm{C}$ and scattered light was collected using an autocorrelator. The particle size of each sample was measured five times. Transmission electron microscopy (TEM) images were obtained with a JEOL TEM-2010HR microscope (JEOL Ltd., Tokyo, Japan) operated at $160 \mathrm{kV}$.

The loading density of the SPIO polymeric micelles was determined using a polarized Zeeman Atomic Absorption Spectrophotometer (Z-2000; Hitachi High Technologies, Tokyo, Japan). Briefly, freeze-dried powders of 5-ml SPIO-loaded nanovesicle solutions were weighed and were combined with $1 \mathrm{M}$ hydrochloric acid $(\mathrm{HCl} ; 10$ wt. \% in water) to accelerate polymer degradation. Complete dissolution of the SPIO was achieved after 3 days. Fe concentrations were detected at $248.3 \mathrm{~nm}$, the absorption wavelength specific for $\mathrm{Fe}$, according to a previously established calibration curve (26). SPIO loading density was calculated as the ratio of the encapsulated SPIO over the total vesicle weight.

Cell culture. Tca-8113 (Institute of Biochemistry and Cell Biology; Chinese Academy of Sciences, Shanghai, China) is a human tongue cancer cell line that overexpresses cell surface receptors for folate. Tca- 8113 cells were grown in Minimal Essential Media (MEM; Invitrogen; Thermo Fisher Scientific, Inc., Waltham, MA, USA) supplemented with $10 \%$ heat-inactivated fetal bovine serum (Invitrogen; Thermo Fisher Scientific, Inc.) and were maintained at $37^{\circ} \mathrm{C}$ in a humidified atmosphere with $5 \% \mathrm{CO}_{2}$.

Biocompatibility assay. Methyl thiazolyl tetrazolium (MTT; Sigma-Aldrich; Merck KGaA) was used to assay the cytotoxicity of folate-targeted SPIO-PEG-PCL and folate-free SPIO-PEG-PCL micelles towards Tca-8113 cells. Briefly, Tca-8113 cells were seeded in $96-$ well plates $\left(1 \times 10^{5}\right.$ cells/well $)$ and the cells were incubated with graded dilutions (1-, 2-, 4-, 8-, 16-, 32-, and 64-fold) of nanovesicle solutions in MEM medium. The plates were incubated at $37^{\circ} \mathrm{C}$ in a humidified atmosphere with $5 \% \mathrm{CO}_{2}$ for 3 days. Subsequently, the folate-targeted SPIO-PEG-PCL micelles were washed twice with phosphate-buffered saline (PBS) and incubated in fresh medium containing $20 \mu \mathrm{l}$ MTT solution in PBS $(5 \mathrm{mg} / \mathrm{ml})$ at $37^{\circ} \mathrm{C}$. After $4 \mathrm{~h}$, the supernatant in each well was discarded and $150 \mathrm{ml}$ dimethyl sulfoxide (Sigma-Aldrich; Merck KGaA) was added to each well to dissolve the formazen. Following gentle agitation of the plates for $10 \mathrm{~min}$, the optical density of each well at a wavelength of $570 \mathrm{~nm}$ was recorded using an Infinite F200 multimode plate reader (Tecan, Mànnedorf, Switzerland). Three duplicates were measured for each experimental sample.

In vitro MRI. Tca- 8113 cells $\left(1 \times 10^{6}\right.$ cells/well) were incubated at $37^{\circ} \mathrm{C}$ with folate-targeted or folate-free SPIO-PEG-PCL micelles at an $\mathrm{Fe}$ concentration of $80 \mu \mathrm{g} / \mathrm{ml}$ in folate-free MEM. Following incubation periods of $0.5,1$, and $2 \mathrm{~h}$ in a humidified atmosphere with $5 \% \mathrm{CO}_{2}$ at $37^{\circ} \mathrm{C}$, the cells were washed with PBS three times, trypsinized, mixed with a $2 \%$ agarose solution in PBS and scanned with a 3.0 T MRI 


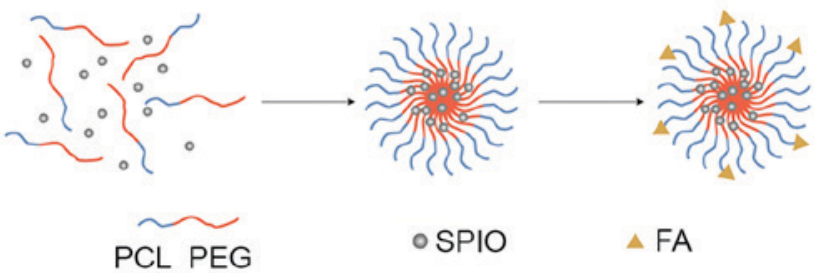

Figure 1. Schematic illustration of folate-targeted SPIO-PEG-PCL micelle formation. SPIO, superparamagnetic iron oxide nanoparticles; PEG, polyethylene glycol; PCL, poly-caprolactone; FA, folic acid

scanner (Magnetom Avanto; version Syngo MR B17, Siemens Healthcare Sector, Erlangen, Germany) at room temperature. A spin echo (SE) $\mathrm{T}_{1}$-weighted sequence [repetition time $\left(\mathrm{T}_{\mathrm{R}}\right)$, $300 \mathrm{msec}$; echo time $\left.\left(\mathrm{T}_{\mathrm{E}}\right), 17 \mathrm{msec}\right]$, a fast spin-echo (FSE) $\mathrm{T}_{2}$-weighted sequence ( $\left.\mathrm{T}_{2} \mathrm{WI} ; \mathrm{TR}, 3,000 \mathrm{msec} ; \mathrm{T}_{\mathrm{E}}, 105 \mathrm{msec}\right)$, and $\mathrm{T}_{2}$ mapping $\left(\mathrm{T}_{\mathrm{R}}, 2,000 \mathrm{msec}, \mathrm{T}_{\mathrm{E}}, 30,60,90\right.$, and $120 \mathrm{msec}$, flip angle, $180^{\circ}$, total duration, $4 \mathrm{~min} 13 \mathrm{sec}$ ) were acquired with the following parameters: Head coil; axial scanning; slice thickness, $2.0 \mathrm{~mm}$; slice spacing, $0.2 \mathrm{~mm}$; field-of-view, 180x180 $\mathrm{mm}^{2}$; and matrix, 256x256. MapIT software (version, Syngo MR B17; Siemens Healthcare Sector) was applied to measure the $\mathrm{T}_{2}$ relaxation time at the work station, with a circular region of interest (ROI; area, $44.9 \mathrm{~mm}^{2}$ ). The shape and area of the ROI were consistent. Relative $\mathrm{T}_{2}$ relaxation time were calculated as follows (26): $\mathrm{T}_{2}=\left[\left(\mathrm{T}^{\prime}-\mathrm{T}_{0}\right) / \mathrm{T}_{0}\right] \times 100 \%$, where $T^{\prime}$ indicates $T_{2}$ relaxation time obtained at different points during incubation and $\mathrm{T}_{0}$ represents the $\mathrm{T}_{2}$ relaxation time prior to incubation.

Prussian blue staining. Tca-8113 cells $\left(5 \times 10^{5}\right.$ cells $\left./ \mathrm{ml}\right)$ were incubated for $1 \mathrm{~h}$ at $37^{\circ} \mathrm{C}$ with folate-targeted and folate-free SPIO-PEG-PCL micelles in folate-free RPMI-1640 medium (Gibco; Thermo Fisher Scientific, Inc.) containing $80 \mu \mathrm{g} / \mathrm{ml} \mathrm{Fe}$ (Gibco; Thermo Fisher Scientific, Inc.; 61870-036). The cells were washed three times with folate-free RPMI-1640 medium and trypsinized (10-5 mol/l). The cell suspensions were subsequently washed with PBS three times before being fixed with $4 \%$ glutaraldehyde. After $10 \mathrm{~min}$, the cells were incubated at $37^{\circ} \mathrm{C}$ with $2 \mathrm{ml}$ Prussian blue solution that was composed of an equal volume of $2 \% \mathrm{HCl}$ acid aqueous solution and $2 \%$ potassium ferrocyanide (II) trihydrate. After $30 \mathrm{~min}$, the cells were briefly stained with $0.5 \%$ neutral red for $3 \mathrm{~min}$. The resulting staining was observed under a light microscope. The intensity of staining was defined by eye with the following scoring system: i) 0 , no staining; ii) +, weak staining; iii) ++, moderate staining; and iv) +++, strong staining (27).

Statistical analysis. Statistical analyses were conducted with SPSS software (version 11.0; SPSS, Inc., Chicago, IL, USA). One-way analysis of variance and a Bonferroni post hoc test were used to identify differences between groups, and $\mathrm{P}<0.05$ was considered to indicate a statistically significant difference.

\section{Results}

Micelle size and biocompatibility. Sizes of the folate-free SPIO-PEG-PCL and folate-targeted SPIO-PEG-PCL micelles were estimated to be 45.5 and $50.2 \mathrm{~nm}$, respectively, according to the DLS measurement (Fig. 2). In the MTT viability assays of the Tca-8113 cells, various SPIO concentrations were evaluated. No statistically significant differences were identified between the folate-free SPIO-PEG-PCL and folate-targeted SPIO-PEG-PCL groups $(\mathrm{F}=0.698 ; \mathrm{P}=0.676)$, thereby demonstrating that increased concentrations of either type of SPIO-PEG-PCL micelle did not increase cytotoxicity (Fig. 3).

In vitro MRI. To validate the tumor targeting ability of the nanoparticles generated, MRI signal intensity was measured for each set of cells that was incubated with folate-targeted SPIO-PEG-PCL and folate-free SPIO-PEG-PCL micelles, respectively. The $\mathrm{T}_{2} \mathrm{WI}$ signal intensity of the two sets of cells decreased marginally after $0.5 \mathrm{~h}$ (Fig. 4), and a time-dependent decrease in signal intensity was observed for the two groups. However, compared with the folate-targeted SPIO-PEG-PCL micelles, the decrease in signal intensity for the folate-free SPIO-PEG-PCL micelles was less marked (Fig. 4). Relative $\mathrm{T}_{2}$ relaxation time was calculated and, under the same conditions, the MRI signals of the cells incubated with the folate-targeted SPIO-PEG-PCL micelles exhibited a significantly greater weakening in signal compared with the folate-free SPIO-PEG-PCL micelles ( $\mathrm{P}=0.002$; Fig. 5).

Prussian blue staining. Entrapment of the SPIO in the nanomicelles allowed direct visualization of their uptake by Tca-8113 cells. As shown in Fig. 6, Prussian blue staining experiments demonstrated that the cells incubated with the folate-targeted SPIO-PEG-PCL micelles exhibited strong staining $(+++)$, which was markedly stronger staining than the cells that were incubated with the folate-free SPIO-PEG-PCL micelles (moderate staining, ++ ).

\section{Discussion}

Advances in imaging technology and their application to cellular biology have brought molecular imaging to the forefront (28). Simultaneously, the identification of molecular targets has been applied to the development and design of various molecular imaging probes for solid tumors (29). As a diagnostic technique, MRI is currently one of the most powerful, non-invasive imaging modalities that is used in clinical medicine today (30), and SPIO represent one of the most effective MRI contrast agents. In the present study, polymeric micelles were generated for the delivery of SPIO, and these SPIO were attached to folate to achieve a targeted-micelle system. The folate receptor is overexpressed by a wide range of malignant cells, including tongue cancer cells. Therefore, the aim of the present study was to combine folate with SPIO-PEG-PCL copolymers to provide tumor-specific imaging following folate receptor-mediated endocytosis of these micelles. In the MRI experiments conducted, this folate-targeted micelle system effectively weakened the MRI signal of Tca-8113 cells. Prussian blue staining further confirmed the endocytosis of the targeted micelles by Tca-8113 cells. The folate-targeted SPIO-PEG-PCL demonstrated potential as a platform for diagnostic imaging applications.

It has been shown that tumor vasculature exhibits poor architecture due to the absence of a pericyte lining and a poor lymphatic drainage system (31). Correspondingly, this 


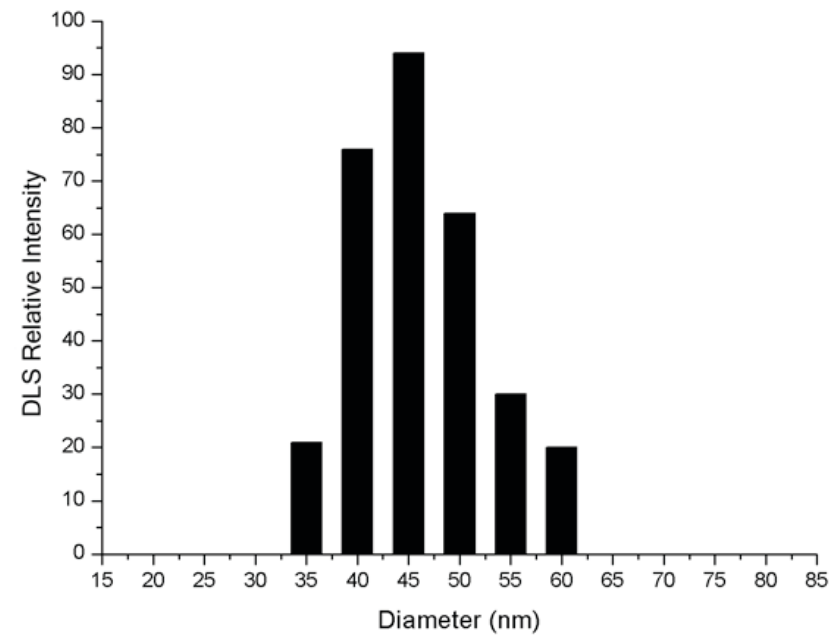

folate-free SPIO-PEG-PCL

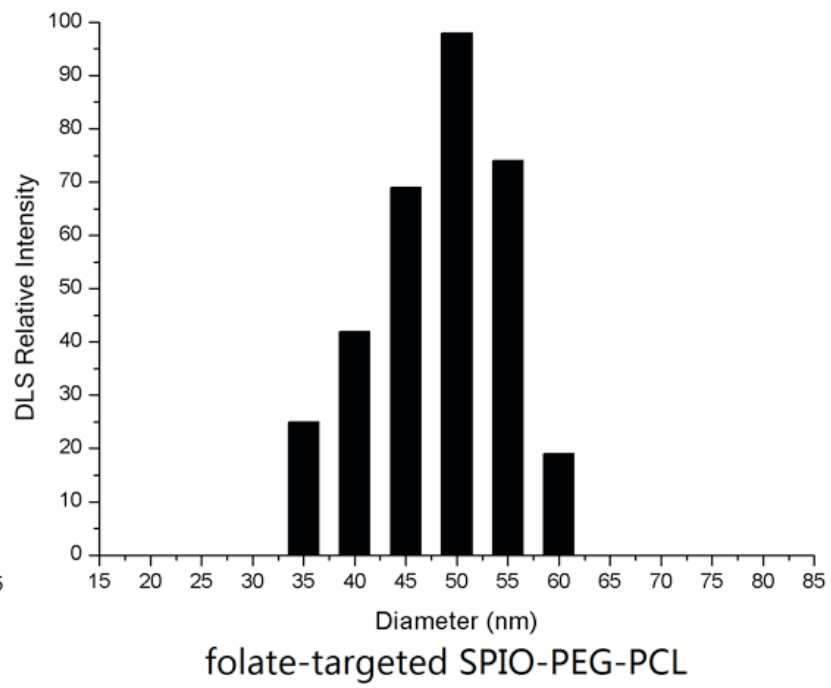

folate-targeted SPIO-PEG-PCL

Figure 2. Size distribution of folate-free SPIO-PEG-PCL and folate-targeted SPIO-PEG-PCL micelles as determined by dynamic light scattering measurements. SPIO, superparamagnetic iron oxide nanoparticles; PEG, polyethylene glycol; PCL, poly-caprolactone.

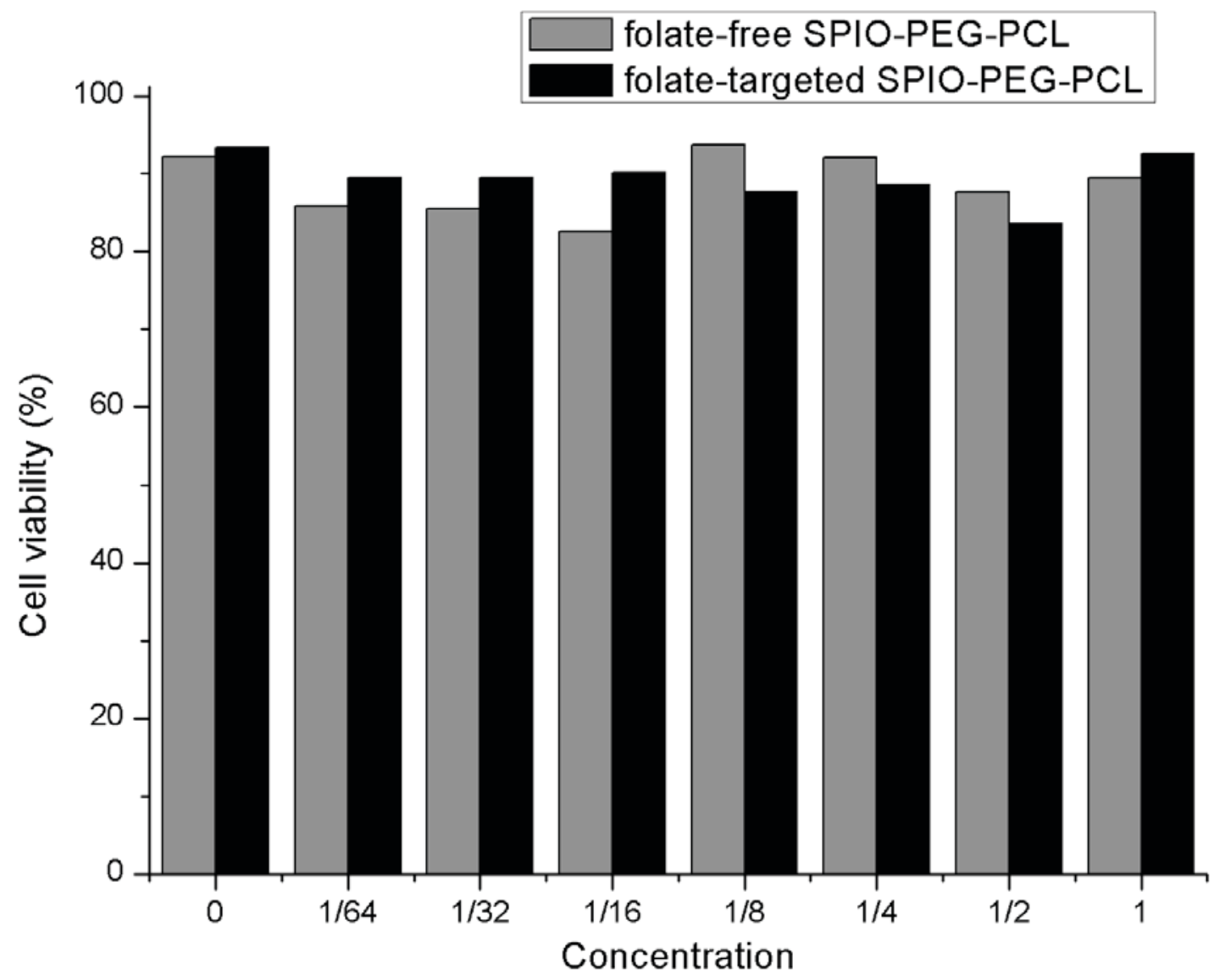

Figure 3. In vitro cytotoxicity of graded dilutions (1-, 2-, 4-, 8-, 16-, 32- and 64-fold) of folate-targeted SPIO-PEG-PCL and folate-free SPIO-PEG-PCL micelles towards Tca-8113 cells, as detected by a methyl thiazolyl tetrazolium assay. SPIO, superparamagnetic iron oxide nanoparticles; PEG, polyethylene glycol; PCL, poly-caprolactone.

leaky vasculature leads to a stronger differential interstitial pressure at the center of tumors when compared with the tumor periphery, and this is referred to as the enhanced permeability and retention (EPR) effect $(31,32)$. For particles ranging in size from 10-100 $\mathrm{nm}$, the EPR effect is consistent with the preferential accumulation of particles in a tumor and extended blood circulation times $(33,34)$. In the present study, DLS measurement demonstrated that the mean diameter for the folate-targeted PEG-PCL-SPIO micelle was $\sim 50.2 \mathrm{~nm}$. This diameter is suitable for efficient targeting in 


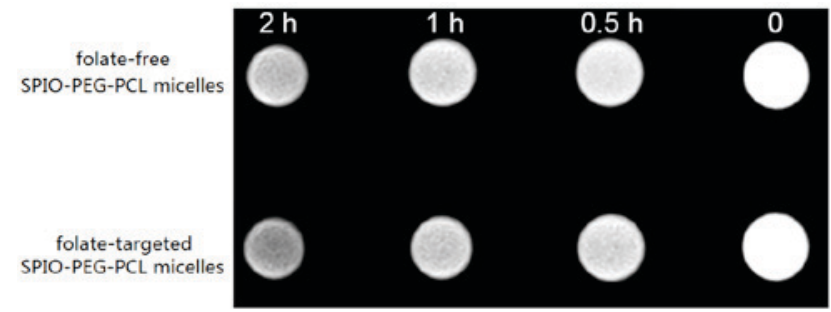

Figure 4. In vitro MRI of the Tca- 8113 cells that were incubated with folate-free SPIO-PEG-PCL and folate-targeted SPIO-PEG-PCL micelles for various incubation durations. The MRI signal intensities of the folate-targeted cells were stronger than those of the cells that were incubated with the folate-free micelles. SPIO, superparamagnetic iron oxide nanoparticles; PEG, polyethylene glycol; PCL, poly-caprolactone; MRI, magnetic resonance imaging.

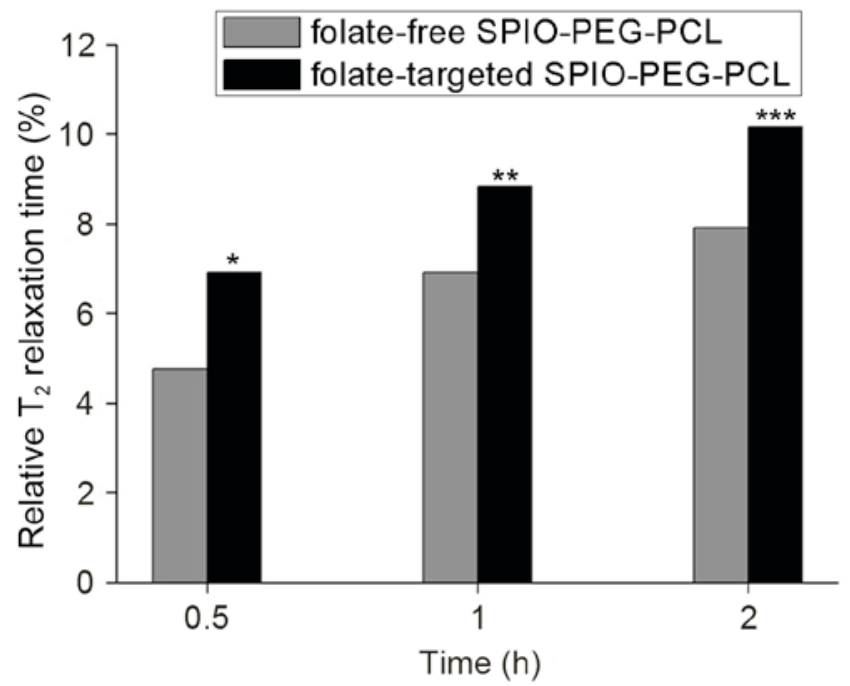

Figure 5. Relative $\mathrm{T}_{2}$ relaxation times of the Tca- 8113 cells that were incubated with the folate-targeted SPIO-PEG-PCL and folate-free SPIO-PEG-PCL micelles. For each incubation period, the MRI signals associated with the folate-targeted SPIO-PEG-PCL micelle treatment were weaker than those of the folate-free SPIO-PEG-PCL micelle treatment. For the whole period, the MRI signals of the cells incubated with the folate-targeted SPIO-PEG-PCL micelles exhibited a significantly greater weakening in signal when compared with the folate-free SPIO-PEG-PCL micelles $(\mathrm{P}=0.002) .{ }^{*} \mathrm{P}<0.05,{ }^{* *} \mathrm{P}=0.01$ and ${ }^{* * *} \mathrm{P}<0.001$ vs. folate-free. SPIO, superparamagnetic iron oxide nanoparticles; PEG, polyethylene glycol; PCL, poly-caprolactone; MRI, magnetic resonance imaging.

MRI applications, it is highly desirable for achieving a longer blood circulation time, and it facilitates the uptake of SPIO by cancer cells (35).

In the present study, MTT assays were performed to evaluate the possible cytotoxicity of the generated micelles and no obvious decrease in cell viability was observed despite the increasing concentrations of the polymers in these assays. Furthermore, when the micelles were incubated in the 1-fold dilution, the level of cytotoxicity detected was considered to be acceptable. Thus, copolymers of the folate-targeted SPIO-PEG-PCL exhibited minimal cytotoxicity towards the Tca-8113 cells examined.

In the MRI conducted in the present study, incubation of the Tca-8113 cells with the folate-targeted SPIO-PEG-PCL micelles resulted in a significant decrease in signal intensity and relative $\mathrm{T}_{2}$ relaxation time. Furthermore, these same

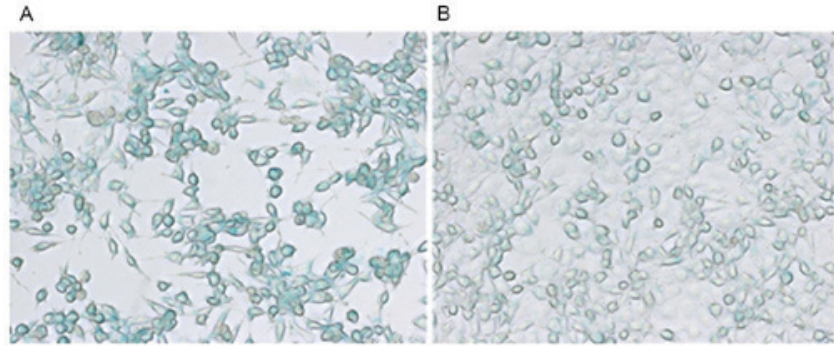

Figure 6. Prussian blue staining of Tca- 8113 cells that were incubated with (A) folate-targeted SPIO-PEG-PCL micelles vs. (B) folate-free SPIO-PEG-PCL micelles for $2 \mathrm{~h}$ (magnification, x200). SPIO, superparamagnetic iron oxide nanoparticles; PEG, polyethylene glycol; PCL, poly-caprolactone.

decreases were not observed for the Tca-8113 cells that were incubated with the folate-free SPIO-PEG-PCL micelles. Consistent with these results, stronger Prussian blue staining was observed for the folate-targeted SPIO-PEG-PCL micelles versus the folate-free SPIO-PEG-PCL micelles, thereby indicating a higher intracellular Fe concentration in the former. Thus, cellular uptake of the micelles appeared to be dependent on the presence of the folate ligand. These results are consistent with those of Yang et al (23) and Hong et al (29), who demonstrated that hydrophobic SPIO nanocrystals that were loaded into folate-targeted SPIO-PEG-PCL micelles provided targeted delivery of an anticancer drug to hepatocellular carcinoma.

Therefore, these folate-targeted SPIO-PEG-PCL micelles demonstrated high sensitivity for MRI. This indicates that this type of micelle has the potential capacity to be an ideal MRI contrast agent for tongue tumors. Furthermore, the results of the Prussian blue staining in the present study revealed that folate-targeted micelles revealed a superior targeting effect on tongue cancer cells than the folate-free micelles. Thus, folate-targeted micelles have a greater potential as a drug delivery system to treat tongue tumors than folate-free micelles.

However, there were two main limitations associated with the present study. The examination of folate targeting was only performed in vitro and was not investigated in vivo. In addition, the delivery efficiency of the micelles was not evaluated using an anti-cancer drug. Thus, future studies will include monitoring of the transfer efficiency of SPIO and anti-cancer drugs to tongue cancers in vivo in order to validate the present findings and to confirm the potential for this method to provide early detection of tongue cancer.

In conclusion, compared with folate-free SPIO-PEG-PCL micelles, folate-targeted SPIO-PEG-PCL micelles exhibited superior targeting for Tca-8113 cells and achieved an ideal imaging effect in vitro MRI. This novel targeted micelle may be used as an effective contrast agent for the early diagnosis of tongue cancer.

\section{Acknowledgements}

The authors received grants from the Natural ScienceFoundation of Guangdong Province (grant no. 2014A030310484) and the S\&T Programs of Guangdong Province (grant no. 2012B031800086). 


\section{References}

1. Hartl DM, Dauchy S, Escande C, Bretagne E, Janot F and Kolb F: Quality of life after free-flap tongue reconstruction. J Laryngol Otol 123: 550-554, 2009.

2. Patel SC, Carpenter WR, Tyree S, Couch ME, Weissler M, Hackman T, Hayes DN, Shores C and Chera BS: Increasing incidence of oral tongue squamous cell carcinoma in young white women, age 18 to 44 years. J Clin Oncol 29: 1488-1494, 2011.

3. Liu Z, Wang H and Li Q: Tongue tumor detection inmedical hyperspectral images. Sensors (Basel) 12: 162-174, 2012.

4. Ong CK and Chong VF: Imaging of tongue carcinoma. Cancer Imaging 6: 186-193, 2006.

5. Chen W, Zhang C, Zhang S, Liang L, Zhang B, Liu C, Zhang Z and Liang C: Application value of MRI combined with positron emission tomography (PET)/CT in diagnosis and preoperative staging of tongue squamous cell carcinoma. J Med Imaging Radiat Oncol 59: 170-178, 2015.

6. Kim BY, Rutka JT and Chan WC: Nanomedicine. N Engl J Med 363: 2434-2443, 2010.

7. Baetke SC, Lammers T and Kiessling F: Applications of nanoparticles for diagnosis and therapy of cancer. Br J Radiol 88: 20150207, 2015

8. Rosen JE, Chan L, Shieh DB and Gu FX: Iron oxide nanoparticles for targeted cancer imaging and diagnostics. Nanomedicine 8: 275-290, 2012

9. Raji MA, Amara M, Amoabediny G, Tajik P, Barin A, Magierowski S and Ghafar-Zadeh E: Cytotoxicity of synthesized iron oxide nanoparticles: Toward novel biomarkers of colon cancer. Conf Proc IEEE Eng Med Biol Soc 2014: 6179-6182, 2014.

10. Landmark KJ, Dimaggio S, Ward J, Kelly C, Vogt S, Hong S, Kotlyar A, Myc A, Thomas TP, Penner-Hahn JE, et al: Synthesis, characterization, and in vitro testing of superparamagneticiron oxide nanoparticles targeted using folic acid-conjugated dendrimers. ACS Nano 2: 773-783, 2008.

11. Hahn MA, Singh AK, Sharma P, Brown SC and Moudgil BM: Nanoparticles as contrast agents for in-vivo bioimaging: Current status and future perspectives. Anal Bioanal Chem 399: 3-27, 2011.

12. Rosen JE, Chan L, Shieh DB and Gu FX: Iron oxide nanoparticles for targeted cancer imaging and diagnostics. Nanomedicine 8 275-290, 2012

13. Barreto JA, O'Malley W, Kubeil M, Graham B, Stephan H and Spiccia L: Nanomaterials: Applications in cancer imaging and therapy. Adv Mater 23: H18-H40, 2011.

14. Hong G, Yuan R, Liang B, Shen J, Yang X and Shuai X: Folate-functionalized polymeric micelle as hepatic carcinoma-targeted, MRI-ultrasensitive delivery system of antitumor drugs. Biomed Microdevices 10: 693-700, 2008.

15. Nasongkla N, Bey E, Ren J, Ai H, Khemtong C, Guthi JS Chin SF, Sherry AD, Boothman DA and Gao J: Multifunctional polymeric micelles as cancer-targeted, MRI-ultrasensitive drug delivery systems. Nano Lett 6: 2427-2430, 2006.

16. Li R, Li X, Xie L, Ding D, Hu Y, Qian X, Yu L, Ding Y, Jiang X and Liu B: Preparation and evaluation of PEG-PCL nanoparticles for local tetradrine delivery. Int J Pharm 379: 158-166, 2009.

17. Pridgen EM, Langer R and Farokhzad OC: Biodegradable, polymeric nanoparticle delivery systems for cancer therapy. Nanomedicine (Lond) 2: 669-680, 2007.

18. Gong C, Shi S, Dong P, Kan B, Gou M, Wang X, Li X, Luo F, Zhao X, Wei Y, et al: Synthesis and characterization of PEG-PCL-PEG thermosensitive hydrogel. Int J Pharm 365: 89-99, 2009.
19. Hong G, Yuan R, Liang B, Shen J, Yang X and Shuai X: Folate-functionalized polymeric micelle as hepatic carcinoma-targeted, MRI-ultrasensitive delivery system of antitumor drugs. Biomed Microdevices 10: 693-700, 2008.

20. Xie M, Zhang H, Xu Y, Liu T, Chen S, Wang J and Zhang T: Expression of folate receptors in nasopharyngeal and laryngeal carcinoma and folate receptor-mediated endocytosis by molecular targeted nanomedicine. Int J Nanomedicine 8: 2443-2451, 2013.

21. Lu Y and Low PS: Immunotherapy of folate receptor-expressing tumors: Review of recent advances and future prospects. J Control Release 91: 17-29, 2003.

22. Kim YK, Minai-Tehrani A, Lee JH, Cho CS, Cho MH and Jiang HL: Therapeutic efficiency of folated poly(ethylene glycol)-chitosan-graft-polyethylenimine-Pdcd4 complexes in $\mathrm{H}-\mathrm{ras} 12 \mathrm{~V}$ mice with liver cancer. Int $\mathrm{J}$ Nanomedicine 8: 1489-1498, 2013

23. Yang X, Deng W, Fu L, Blanco E, Gao J, Quan D and Shuai X: Folate-functionalized polymeric micelles for tumor targeted delivery of a potent multidrug-resistance modulator FG020326. J Biomed Mater Res A 86: 48-60, 2008.

24. Shuai X, Ai H, Nasongkla N, Kim S and Gao J: Micellar carriers based on block copolymers of poly(epsilon-caprolactone) and poly(ethylene glycol) for doxorubicin delivery. J Control Release 98: 415-426, 2004.

25. Sun S, Zeng H, Robinson DB, Raoux S, Rice PM, Wang SX and Li G: Monodisperse MFe2O4 (M=Fe, Co, Mn) nanoparticles. J Am Chem Soc 126: 273-279, 2004

26. Feng ST, Li J, Luo Y, Yin T, Cai H, Wang Y, Dong Z, Shuai X and Li ZP: pH-Sensitive nanomicelles for controlled and efficient drug delivery to human colorectal carcinoma LoVo cells. PloS One 9: e100732, 2014

27. Prom-u-thai C, Dell B, Thomson G and Rerkasem B: Easy and rapid detection of iron in rice grain. ScienceAsia 29: 203-207, 2003.

28. Margolis DJ, Hoffman JM, Herfkens RJ, Jeffrey RB, Quon A and Gambhir SS: Molecular imaging techniques in body imaging. Radiology 245: 333-356, 2007.

29. Hong GB, Zhou JX and Yuan RX: Folate-targeted polymeric micelles loaded with ultrasmall superparamagnetic iron oxide: Combined small size and high MRI sensitivity. Int J Nanomedicine 7: 2863-2872, 2012.

30. Bautista MC, Bomati-Miguel O, Zhao X, Morales MP, Gonzalez-Carreno T, Perez de Alejo R, Ruiz-Cabello J and Veintemillas-Verdaguer S: Comparative study of ferrofluids based on dextran-coated iron oxide and metal nanoparticles for contrast agents in magnetic resonance imaging. Nanotechnology 15: $154-159,2004$.

31. Maeda H, Nakamura H and Fang J: The EPR effect for macromolecular drug delivery to solid tumors: Improvement of tumor uptake, lowering of systemic toxicity, and distinct tumor imaging in vivo. Adv Drug Deliv Rev 65: 71-79, 2013.

32. Greish K: Enhanced permeability and retention of macromolecular drugs in solid tumors: A royal gate for targeted anticancer nanomedicines. J Drug Target 15: 457-464, 2007.

33. Byrne JD, Betancourt T and Brannon-Peppas L: Active targeting schemes for nanoparticle systems in cancer therapeutics. Adv Drug Deliv Rev 60: 1615-1626, 2008.

34. Ranganathan R, Madanmohan S, Kesavan A, Baskar G, Krishnamoorthy YR, Santosham R, Ponraju D, Rayala SK and Venkatraman G: Nanomedicine: Towards development of patient-friendly drug-delivery systems for oncological applications. Int J Nanomedicine 7: 1043-1060, 2012.

35. Lee JH, Huh YM, Jun YW, Seo JW, Jang JT, Song HT, Kim S, Cho EJ, Yoon HG, Suh JS, et al: Artificially engineered magnetic nanoparticles for ultra-sensitive molecular imaging. Nat Med 13: 95-99, 2007. 